\title{
Editorial
}

Z Gerontol Geriat 2019· 52:307-308

https://doi.org/10.1007/s00391-019-01564-w

(c) Springer Medizin Verlag GmbH, ein Teil von Springer Nature 2019

\author{
L. C. Bollheimer' ·. M. Bauer' ' W. Hofmann ${ }^{3}$ \\ 'Lehrstuhl für Altersmedizin mit Klinik für Innere Medizin und Geriatrie am Franziskushospital Aachen, \\ Uniklinik RWTH Aachen, Aachen, Deutschland \\ ${ }^{2}$ Geriatrisches Zentrum, Universität Heidelberg und Agaplesion Bethanien Krankenhaus, Heidelberg, \\ Deutschland \\ ${ }^{3}$ Rechlin, Deutschland
}

\section{Grammatik der Neurogeriatrie}

Liebe Leser,

Sie finden in diesem Heft „Neurogeriatrie“ vier Themenschwerpunktbeiträge, die ausgehend von den typischen neurologischen Befunden der Bewegungsstörung, Balancestörung, Kognitionseinbuße und Dysphagie organübergreifend Aspekte der Funktionalität, Autonomie und Lebensqualität bei alten Patienten behandeln, also „klassisch geriatrisch“ ausgearbeitet sind.

Weshalb diese Vorbemerkung? Sie sollten über die Hintergründe informiert sein: Die Politik (Kuratorium Deutsche Altershilfe und WilhelmineLübke-Stiftung) hatte 1973 den ersten Lehrstuhl für Altersmedizin in Erlangen gestiftet. Heute halten die im Medizinischen Fakultätentag (MFT) zusammengeschlossenen 38 medizinischen Fakultäten in Deutschland 11 Lehrstühle resp. Professuren für Geriatrie vor, wovon 10 internistisch und eine neurologisch geprägt sind. Sieben weitere Ausschreibungen bzw. Berufungsverfahren sind aktuell im Gange.

Auf der nichtwissenschaftlichen, d.h. institutionellen, Klinikebene bilden sich zunehmend häufiger interdisziplinäre geriatrische Zentren unter Mitwirkung der inneren Medizin, der Unfallchirurgie, der Neurologie und auch der Gerontopsychiatrie, dabei mit lokal deutlich unterschiedlicher Akzentuierung.

Droht hier eine Aufsplitterung der Geriatrie? Sprachlich könnte man es meinen, denn auch der Gebrauch von „geriatrischen Determinativkomposita“, wie auch im vorliegenden Titel „Neurogeriatrie“, nimmt im deutschen wie auch im internationalen Schrifttum zu.
Vor diesem Hintergrund führte der Begriff „Neurogeriatrie“ im Herausgeberkreis dieser Zeitschrift zu Diskussionen, die auch (fach-)politisch geprägt waren. Denn die Konzeptionalisierung einer Neurogeriatrie steht in Deutschland sowohl auf der wissenschaftlichen als auch auf der institutionellen Agenda. Allerdings versteht sich die Zeitschrift für Gerontologie und Geriatrie als interdisziplinäres, multiprofessionelles (Psychologie, Soziologie, Biologie, Rechtswissenschaft u. v. a. m.) und v. a. wissenschaftliches Publikationsorgan.

Bei den oben erwähnten geriatrischen Determinativkomposita wird ein klinisches Fachgebiet als Bestimmungswort dem geriatrischen Grundwort vorangestellt („Neurogeriatrie“, „orthogeriatrics“, "psychogeriatrics“...). Ist dadurch schon wortschöpferisch die Geriatrie hintangestellt? Und erhebt sich nicht auch umgekehrt sofort die Frage nach dem Koch oder Kellner, wenn ein klinisches Fachgebiet das Grundwort für sich selbst beansprucht und die Geriatrie nur noch im altersspezifizierenden Bestimmungswort resp. Adjektiv vorkommt („Alterstraumatologie“, „Gerontopsychiatrie“, "geriatric oncology“, "geriatric oncology“...)?

Derartige Überinterpretationen der semantischen Relation sind müßig, denn die geriatrischen Determinativkomposita sollen wissenschaftlich nichts anderes sein als der Aufruf zur Interprofessionalität, welche die Geriatrie für den gegenwartsnahen Ausbau ihres längst nicht abgeschlossenen fachlichen Fundaments dringend benötigt.

Das Bemühen der Geriaterin und des Geriaters um ein breitgefächertes Wissen (Supraspezialität) bringt es mit sich, häu- 


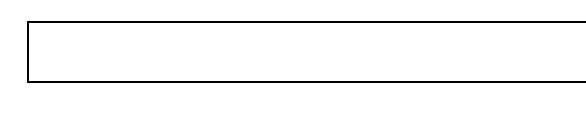

figer Fragende und nicht Gefragte zu sein. Hier bedarf es für die Weiterentwicklung des Fachs zahlreicher „Wanderer zwischen beiden Welten“, die insbesondere aus den großen Fachgebieten der inneren Medizin, Allgemeinmedizin, Neurologie und Psychiatrie die für die Geriatrie relevanten Einzelaspekte exzerpieren und dann geriatrisch, d. h. unter den Rahmenbedingungen der geriatrischen Fachspezifität (holistische Sichtweise, Funktionsorientiertheit, Multimorbidität), weiterentwickeln. Genau dieses interprofessionelle Prinzip wird im vorliegenden Themenschwerpunkt mit seinen neurogeriatischen „Up-to-date“-Beiträgen zu Bewegungsstörungen, Balance, Dysphagie und natürlich auch Demenz umgesetzt.

Die also mit den geriatrischen Determinativkomposita einhergehende translationale Kernaufgabe betrifft weniger den klinischen Alltag und die Krankenversorgung. Sie rechtfertigt innerhalb des (noch) überschaubaren Kreises der geriatrischen Fachgemeinschaft im deutschsprachigen Raum auch keine eigenständige Subfachlichkeit. Im Sinne einer harmonischen Weiterentwicklung der Geriatrie handelt es sich vielmehr um einen internationalen akademischen Auftrag für die geriatrischen Fachgesellschaften, dem die Geriatrie auch in Deutschland als ein zunehmend universitär ernst genommenes Fach gerecht werden muss.

Genießen Sie die Lektüre und beteiligen Sie sich am Diskurs!

Ihre

Prof. Dr. med. C. Bollheimer,

Prof. Dr. med. J.M. Bauer,

PD Dr. med. W. Hofmann

\section{Korrespondenzadresse}

Prof. Dr. med. L. C. Bollheimer

Lehrstuhl für Altersmedizin mit Klinik für Innere Medizin und Geriatrie am Franziskushospital

Aachen, Uniklinik RWTH Aachen

Morillenhang 27, 52074 Aachen, Deutschland cbollheimer@ukaachen.de

Interessenkonflikt. L. Bollheimer, J. Bauer und W. Hofmann geben an, dass kein Interessenkonflikt besteht.
Lesetipp

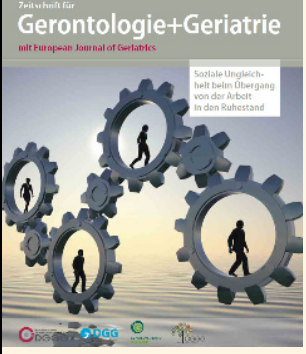

\section{Soziale Ungleichheit beim Übergang von der Arbeit in den Ruhestand}

Neues Online-Supplement der Zeitschrift für Gerontologie und Geriatrie H. M. Hasselhorn, N. Dragano, D. Hofäcker, M. Wahrendorf (Hrsg.)

Die politische Diskussion zur Regulierung von Wegen und Zeitpunkten des Erwerbsaustritts wird in den kommenden Jahren weiter anhalten.

Interessanterweise werden Fragen der sozialen Ungleichheit beim Übergang von der Arbeit in den Ruhestand in der wissenschaftlichen Literatur nur selten explizit thematisiert.

Das NRW-Forschungsnetzwerk „Arbeitsmarktteilhabe im höheren Erwerbsalter“ kam daher überein, ein wissenschaftliches Schwerpunktheft zu initiieren.

Die in diesem Heft vorliegenden Beiträge bestätigen nicht nur die Breite und Vielfalt der Thematik, sondern verdeutlichen, dass die vielen beteiligten Disziplinen wie die Arbeitswissenschaft, Sozialepidemiologie, Gerontologie, Psychologie und Soziologie, jeweils originelle und sich ergänzende Sichtweisen - von der individuellen bis zur strukturell-politischen Ebene - beizusteuern haben. Unter Nutzung hochwertiger Daten betrachten sie unterschiedliche Aspekte horizontaler sozialer Ungleichheit (z. B. Geschlecht, Migrationsstatus, Wirtschaftssektor, Nation) und auch vertikaler sozialer Ungleichheit (beruflicher Status, Einkommen). Dabei verbindet sie ihr jeweiliger Fokus auf die Unterschiedlichkeit der Rahmenbedingungen, des Erlebens und des Verhaltens der betrachteten sozialen Gruppen bei ihrem Übergangsprozess von der Arbeit in den Ruhestand.

Der Wunsch der Herausgeber ist es, in Zeiten immer länger werdender Erwerbsbiografien dazu beizutragen, dass sich die Diskussion zu Ruhestandsübergängen in Öffentlichkeit und Politik nicht zu sehr auf die Frage des (möglichst späten) Zeitpunkts des Erwerbsausstiegs beschränkt. Stattdessen sollte ebenso die angemessene Lebens- und Arbeitsqualität aller älteren Menschen während dieser Übergangsphase diskutiert werden und damit auch Fragen der Chancengleichheit, Gerechtigkeit und auch Würde in einer entscheidenden Lebensphase. Die hier zusammengestellten Beiträge zeigen, dass die Wissenschaft hier ihren Beitrag leisten kann:

- Soziale Ungleichheit beim Übergang von der Arbeit in den Ruhestand

- Vertikale und horizontale soziale Ungleichheit und Motivation zum vorzeitigen Erwerbsausstieg

- Wie erfolgreich sind ältere Arbeitskräfte in der zeitlichen Umsetzung ihrer Ausstiegspläne?

- Transition to retirement in the healthcare sector

- Gender differences in paid employment after retirement

- Wandel von Ruhestandsübergängen im politischen Paradigmenwechsel Europas

- Social inequality in working life expectancy in Sweden

- Soziale Ungleichheiten der Erwerbsminderung bei älteren Arbeitnehmern

- Inanspruchnahme medizinischer Rehabilitation im Vorfeld der Erwerbsminderungsrente

- Consequences of interrupted employment careers on early retirement and resulting pensions entitlements

- Soziale Ungleichheit und Gesundheit im höheren Lebensalter

Abonnenten der Zeitschrift haben kostenfreien Zugriff auf die Inhalte des Supplements

und finden dieses im Online-Archiv der Zeitschrift (Jahrgang 2019) unter:

www.springermedizin.de/zeitschrift-fuer-gerontologie-und-geriatrie 